\author{
Chenggang $\mathrm{Li}$ \\ Yan Wang \\ Jing Chen \\ Jingjing You \\ Hemant Rajnathsing
}

https://doi.org/10.21278/TOF.42307

ISSN 1333-1124

eISSN 1849-1391

\title{
FAULT DIAGNOSIS IN A GYROSCOPE-BASED SIX-AXIS ACCELEROMETER
}

\begin{abstract}
Summary
An investigation into the fault diagnosis of a six-axis accelerometer is of great significance because of the high reliability requirement in areas such as aerospace. The working principle and decoupling algorithm of a six-axis accelerometer are introduced. The six-axis accelerometer and the gyroscope form the measurement system and provide the basis for fault diagnosis and restoration by deriving force and deformation compatibility equations. Descartes three-dimensional coordinate system of fault diagnosis is put forward, which carries out the function of real-time diagnosis of the measurement system. In view of a specific fault case, restoration was performed by replacing fault data of a branch chain with correct data. A relevant experiment was carried out and the results confirm the effectiveness of the restoration.
\end{abstract}

Key words: $\quad$ six-axis accelerometer, gyroscope, fault diagnosis, restoration

\section{Introduction}

Measurement requirements are increasingly demanding resulting in extensive investigations being conducted into six dimensional motion parameters of an object [1]. In recent years, the strict requirements imposed on the six-axis accelerometer as an important instrument that measures the state of motion of an object are getting more and more prominent [2]. For improving the reliability of the measurement system, it is necessary to develop a fault diagnosis and fault restoration system [3]. At present, research on the six-axis accelerometer is in the exploratory stage while fault diagnosis and fault restoration systems are in their infancy.

The proposed fault diagnosis system can be roughly divided into two categories by train of thought. The first category is based on redundant information, including hardware redundancy and information redundancy. Zhao et al. of the Tsinghua University made use of a multiple sensor failure detection method based on a hardware redundancy system to determine which sensor fails [4]. Obviously, this method sets a clear way of thinking, exhibiting both high diagnosis accuracy and system stability. However, the method is cost prohibitive and at the same time the use of multiple sensors can also make the system 
complicated. D.W. Clark and P.M.A. Fraher of the University of Oxford achieved fault detection and correction capability of the permeable membrane plugging which can easily be seen in a dissolved oxygen sensor on the basis of an analysis of a physical diffusion model of oxygen in the system [5]. The second category is based on signal processing, such as correlation analysis and wavelet transform method, using absolute value inspection and trend inspection to judge whether the fault happens. The most common are the expert system and the Artificial Neural Network (ANN). Niaki, Abbasi et al. took advantage of the neural network to identify abnormal variables in a multivariate control chart, in order to achieve the system fault detection [6]. Jian-Da Wu, Chiu-Hong Liu et al. effectively improved the effectiveness of the average classification, with the help of continuous wavelet and discrete wavelet as mother wavelet to build and execute the wireless power transfer (WPT) technology [7]. This method can carry out a real-time, high sensitivity test, but there is still a delay on larger scales. Meanwhile, in spite of the strong fault-tolerant capability and high reliability in fault diagnosis, the complex learning mode, specific logical relations of neural network and expert system, make the contribution of this method limited.

This paper introduces a new kind of six-axis accelerometer which carries out the realtime measurement of six dimensional acceleration of an object. Section 2 gives configurations of the six-axis accelerometer, and the decoupling mechanism is presented. A gyroscope and the accelerometer form the redundant measurement system [8]. The investigation was performed on the fault diagnosis and restoration of the measurement system, and the reliability of the measurement system was improved effectively, which is the focus of Section 3. Simulation and experimental verification were carried out, and the verification result shows that the fault diagnosis and restoration method is feasible.

\section{Introduction of a six-axis accelerometer}

\subsection{Decoupling principle of the six-axis accelerometer}

This paper uses the six-axis accelerometer which is proposed by LI Cheng-gang, YOU Jing-jing et al. [9], whose 3D model and main components are shown in Fig. 1. The six-axis accelerometer utilizes a parallel mechanism [10] to function as an elastomer structure and some piezoelectric ceramics [11] to function as sensitive elements. The accelerometer has high decoupling precision and good real-time performance.

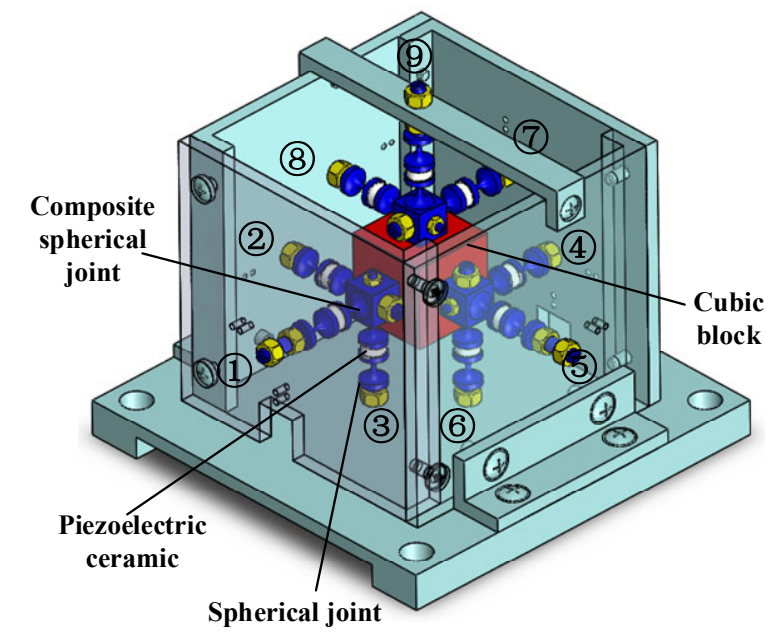

Fig. 1 Model of parallel type six-axis accelerometer

The coordinate system marked $\{\mathrm{M}\}$ and the inertial coordinate system marked $\{\mathrm{O}\}$ are built on a cubic block. In the working process of the six-axis accelerometer, the resultant 
forces that act on the cubic block marked $\boldsymbol{F}^{O}$ and the joint torques that act on the cubic block marked $\boldsymbol{T}^{O}$ are as follows:

$$
\begin{aligned}
& \boldsymbol{F}^{O}=\boldsymbol{A}_{M}^{O}\left[\begin{array}{c}
-f_{3}-f_{6}+f_{9} \\
-f_{2}+f_{5}-f_{8} \\
f_{1}-f_{4}-f_{7} \\
0
\end{array}\right]+\left[\begin{array}{c}
m g \\
0 \\
0 \\
0
\end{array}\right] \\
& \boldsymbol{T}^{O}=n \boldsymbol{A}_{M}^{O}\left[\begin{array}{c}
-f_{2}+f_{4} \\
f_{3}-f_{7} \\
-f_{6}+f_{8} \\
0
\end{array}\right]
\end{aligned}
$$

where $f_{i}$ is the force of the branch chain marked $i, m$ is the mass of the cubic block, $n$ is half of the length of the edge of the cubic block, $g$ is the gravitational acceleration, $A_{M}^{O}$ is the $4 \times 4$ rotation matrix that represents $\{\mathrm{M}\}$ relative to $\{\mathrm{O}\}[12]$.

The system dynamic equations are set up according to the Newton-Euler method; the absolute linear acceleration of the center of the cubic block marked $\boldsymbol{a}^{O}$ and the absolute angular acceleration of the cubic block marked $\varepsilon^{O}$ are as follows:

$$
\begin{aligned}
& \boldsymbol{a}^{O}=\frac{1}{m} \boldsymbol{A}_{M}^{O}\left[\begin{array}{c}
-f_{3}-f_{6}+f_{9} \\
-f_{2}+f_{5}-f_{8} \\
f_{1}-f_{4}-f_{7} \\
0
\end{array}\right]+\left[\begin{array}{l}
g \\
0 \\
0 \\
0
\end{array}\right] \\
& \varepsilon^{O}=\frac{3}{2 m n} \boldsymbol{A}_{M}^{O}\left[\begin{array}{c}
-f_{2}+f_{4} \\
f_{3}-f_{7} \\
-f_{6}+f_{8} \\
0
\end{array}\right]
\end{aligned}
$$

Eqs.(3) and (4) are nonlinear and strongly coupled, so they cannot be directly solved [13]. The auxiliary angular velocity is introduced, marked $\omega^{*}$, and $\omega^{*}$ and $\omega^{O}$, the absolute angular velocity of the cubic block, are set to satisfy the following equations:

$$
\left[\begin{array}{c}
\boldsymbol{\omega}^{O} \\
0
\end{array}\right]=\boldsymbol{A}_{M}^{O}\left[\begin{array}{c}
\boldsymbol{\omega}^{*} \\
0
\end{array}\right]
$$

Meanwhile, based on the improved Euler algorithm [14], the auxiliary angular velocity at the $N^{\text {th }}$ instant also satisfies the following Eq. (6):

$$
\omega_{N}^{*}=h *\left(\sum_{i=1}^{N-1} \varepsilon_{i}^{*}+\frac{\varepsilon_{1}^{*}+\varepsilon_{N}^{*}}{2}\right)
$$

where $h$ is the step length and $\varepsilon_{i}^{*}$ is the auxiliary angular acceleration at the $i^{\text {th }}$ instant which satisfies the following equations:

$$
\left[\begin{array}{c}
\varepsilon_{i} \\
0
\end{array}\right]=\boldsymbol{A}_{M}^{O}\left[\begin{array}{c}
\varepsilon_{i}^{*} \\
0
\end{array}\right]
$$

Solving Eqs.(3) and (4) with the aid of $\omega^{*}$ can yield dynamic equations decoupled completely and result in the six dimensional acceleration. 


\subsection{Force and deformation compatibility equations of the six-axis accelerometer}

It can be seen from Fig. 1 that three composite spherical joints are rigidly connected to the cubic block, which means that the relative distances of the center points of the joints are kept constant. Put $\boldsymbol{b}_{i}$ as the location coordinates of the composite spherical joints, the constraint can be described by the following equations:

$$
\left\{\begin{array}{l}
\left|\boldsymbol{b}_{1}-\boldsymbol{b}_{2}\right|=\sqrt{2} n \\
\left|\boldsymbol{b}_{1}-\boldsymbol{b}_{3}\right|=\sqrt{2} n \\
\left|\boldsymbol{b}_{2}-\boldsymbol{b}_{3}\right|=\sqrt{2} n
\end{array}\right.
$$

Using the forward kinematics theory of the parallel mechanism [15], the coordinate matrix of the composite spherical joints about the forces from $f_{1}$ to $f_{9}$ is derived as the following equations:

$$
\left(\boldsymbol{b}_{1}, \boldsymbol{b}_{2}, \boldsymbol{b}_{3}\right) \approx\left[\begin{array}{ccc}
-f_{3} / k_{3} & -f_{6} / k_{6} & f_{9} / k_{9}-n \\
-f_{2} / k_{2} & f_{5} / k_{5}-n & -f_{8} / k_{8} \\
f_{1} / k_{1}-n & -f_{4} / k_{4} & -f_{7} / k_{7}
\end{array}\right]
$$

where $k_{i}$ is the stiffness coefficient of the piezoelectric ceramic in the branch chain marked $i$.

Substituting Eq. (9) into Eq. (8) and neglecting higher order small values, three force and deformation compatibility equations about the input features are obtained:

$$
\left\{\begin{array}{l}
\frac{f_{1}}{k_{1}}+\frac{f_{2}}{k_{2}}+\frac{f_{4}}{k_{4}}+\frac{f_{5}}{k_{5}} \approx 0 \quad(a) \\
\frac{f_{1}}{k_{1}}+\frac{f_{3}}{k_{3}}+\frac{f_{7}}{k_{7}}+\frac{f_{9}}{k_{9}} \approx 0 \quad(b) \\
\frac{f_{5}}{k_{5}}+\frac{f_{6}}{k_{6}}+\frac{f_{8}}{k_{8}}+\frac{f_{9}}{k_{9}} \approx 0 \quad(\mathrm{c})
\end{array}\right.
$$

Eq. (10) represents the inherent constraints among the system inputs, which are always satisfied under normal working condition and will not change as motion characteristics of the carrier change. So, Eq. (10) can be regarded as the basic fault judgment equation of a six-axis accelerometer. When some or all of the equations are not satisfied, this should be alerted in the system at once and a subsequent analysis and restoration should be conducted.

\section{Six dimensional acceleration measurement system based on gyroscope}

\subsection{Fault diagnosis}

Bring a gyroscope into the original measurement system and assume the gyroscope to be a precision instrument whose measured values are the theoretical values. Now, the input values of the measuring system consist of two kinds, one kind are values of the forces of the branch chains of the six-axis accelerometer and the other kind is the value of angular velocity of the gyroscope.

By further analysis, it is obvious that the input of the gyroscope is just the auxiliary angular velocity mentioned above. Write the output of the gyroscope as $\omega_{N}{ }^{*}=\left[\omega x_{N}{ }^{*} \omega y_{N}{ }^{*} \omega z_{N}{ }^{*}\right]^{T}$, and the derivative of $\omega_{N}^{*}$ as $\varepsilon^{*} . \varepsilon^{*}$ yields solution by means of the numerical differentiation [16] or the differential tracker [17]. Suppose $\varepsilon^{*}$ is a known quantity solved according to $\omega_{N}^{*}$.

Combining Eq. (4) with Eq. (6), we get: 


$$
\left\{\begin{array}{l}
\omega x_{N}{ }^{*}=\sum_{i=1}^{N-1}\left(-f_{2_{i}}+f_{4_{i}}\right)+\frac{-f_{2_{1}}+f_{4_{1}}-f_{2_{N}}+f_{4_{N}}}{2}(d) \\
\omega y_{N}{ }^{*}=\sum_{i=1}^{N-1}\left(f_{3_{i}}-f_{7_{i}}\right)+\frac{f_{3_{1}}-f_{7_{1}}+f_{3_{N}}-f_{7_{N}}}{2} \\
\omega z_{N}{ }^{*}=\sum_{i=1}^{N-1}\left(-f_{6_{i}}+f_{8_{i}}\right)+\frac{-f_{6_{1}}+f_{8_{1}}-f_{6_{N}}+f_{8_{N}}}{2}(f)
\end{array}\right.
$$

where $f_{j_{i}}$ is the force of the branch chain marked $j$ at the $i^{\text {th }}$ instant.

Combining Eq. (4) with Eq. (7), the equations can be written as:

$$
\left\{\begin{array}{l}
-f_{2}+f_{4}=\frac{2 m n}{3} \varepsilon_{x}^{*}(g) \\
f_{3}-f_{7}=\frac{2 m n}{3} \varepsilon_{y}^{*}(h) \\
-f_{6}+f_{8}=\frac{2 m n}{3} \varepsilon_{z}^{*}(i)
\end{array}\right.
$$

Combining Eq. (10) with Eq. (12) further, the force expressions are solved as follows:

$$
\left\{\begin{array}{l}
f_{2} \approx-\frac{2 m n}{3} \cdot \frac{k_{2}}{k_{2}+k_{4}} \varepsilon_{x}^{*}-\left(\frac{f_{1}}{k_{1}}+\frac{f_{5}}{k_{5}}\right) \cdot \frac{k_{2} \cdot k_{4}}{k_{2}+k_{4}} \quad(j) \\
f_{4} \approx \frac{2 m n}{3} \cdot \frac{k_{4}}{k_{2}+k_{4}} \varepsilon_{x}^{*}-\left(\frac{f_{1}}{k_{1}}+\frac{f_{5}}{k_{5}}\right) \cdot \frac{k_{2} \cdot k_{4}}{k_{2}+k_{4}} \quad(k) \\
f_{3} \approx \frac{2 m n}{3} \cdot \frac{k_{3}}{k_{3}+k_{7}} \varepsilon_{y}^{*}-\left(\frac{f_{1}}{k_{1}}+\frac{f_{9}}{k_{9}}\right) \cdot \frac{k_{3} \cdot k_{7}}{k_{3}+k_{7}} \quad(l) \\
f_{7} \approx-\frac{2 m n}{3} \cdot \frac{k_{7}}{k_{3}+k_{7}} \varepsilon_{y}^{*}-\left(\frac{f_{1}}{k_{1}}+\frac{f_{9}}{k_{9}}\right) \cdot \frac{k_{3} \cdot k_{7}}{k_{3}+k_{7}}(m) \\
f_{6} \approx-\frac{2 m n}{3} \cdot \frac{k_{6}}{k_{6}+k_{8}} \varepsilon_{z}^{*}-\left(\frac{f_{5}}{k_{5}}+\frac{f_{9}}{k_{9}}\right) \cdot \frac{k_{6} \cdot k_{8}}{k_{6}+k_{8}} \quad(n) \\
f_{8} \approx \frac{2 m n}{3} \cdot \frac{k_{8}}{k_{6}+k_{8}} \varepsilon_{z}^{*}-\left(\frac{f_{5}}{k_{5}}+\frac{f_{9}}{k_{9}}\right) \cdot \frac{k_{6} \cdot k_{8}}{k_{6}+k_{8}} \quad(o)
\end{array}\right.
$$

Eq. (13) shows further inherent constraint relations among the system inputs after bringing them in the gyroscope. The forces between the branch chains of the six-axis accelerometer satisfy the constraint relations shown in Eq. (10). Inputs of the gyroscope and the six-axis accelerometer satisfy the constraint relations shown in Eqs. (11), (12) and (13). Also, these constraint relations are always satisfied.

In the process of actual measurement, determine whether a failure occurs in the measurement system by judging whether Eqs. (11), (10), (12) and (13) are satisfied entirely. The diagnostic scheme is shown in Fig. 2.

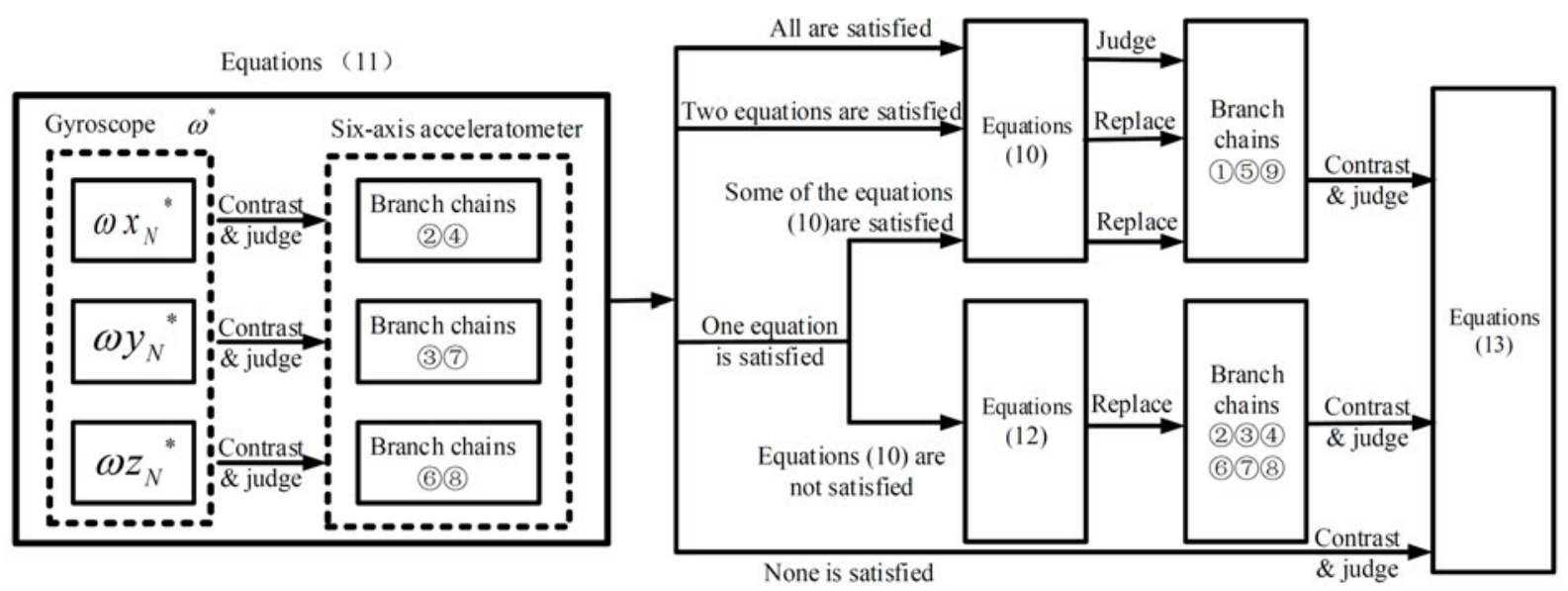

Fig. 2 The diagnostic scheme 
Take note that, for example, Eq. $(d)$ and $(e)$ in Eq. (11) and Eq. (c) in Eq. (10) are not satisfied while Eq. (f) in Eq. (11) is satisfied; introduce the fault diagnosis method specifically.

First of all, put forward a kind of the Descartes three-dimensional coordinate system of fault diagnosis as shown in Fig. 3. The $\mathrm{X}$ axis denotes the way of substitution; that is, calculate the forces of the branch chains (5) and (9) with the aid of Eq. (c) and put the results into Eq. (13) to calculate theoretical values of the branch chains. Two conditions are denoted in the $\mathrm{X}$ axis in the front view of Fig. 3, which are the branch chains (5) to (9) and the branch chains (9) to (5). From the $\mathrm{Y}$ axis it can be judged whether the actual measured values of the branch chains (2) and (4) match the theoretical values provided by Eq. $(j)$ and $(k)$ in Eq. (13).

From the $\mathrm{Z}$ axis it can be judged whether the actual measured values of the branch chains (3) and (7) match the theoretical values provided by Eq. $(l)$ and $(m)$ in Eq. (13). If the former matches and the latter does not, mark it as ' $\mathrm{YN}$ '; if the latter matches and the former does not, mark it as 'NY'; if both do not match, mark it as ' $\mathrm{NN}$ '. Both branch chains cannot match since Eq. $(d)$ and Eq.(e) in Eq.(11) are not established; which explains that at least one of the branch chains (2) and (4) should break down, and at least one of the branch chains (3) and 7 should break down. Mark the numbers of specific fault branch chains diagnosed in the corresponding cubes while the undiagnosed branch chains and the system failure are shown in grey cubes.

Second, Eq. $(f)$ is satisfied while Eq. $(c)$ is not, which explains that at least one of the branch chains (5) and (9) should break down. Meanwhile, the failure possibility of the branch chains (6) and (8) can be ruled out.

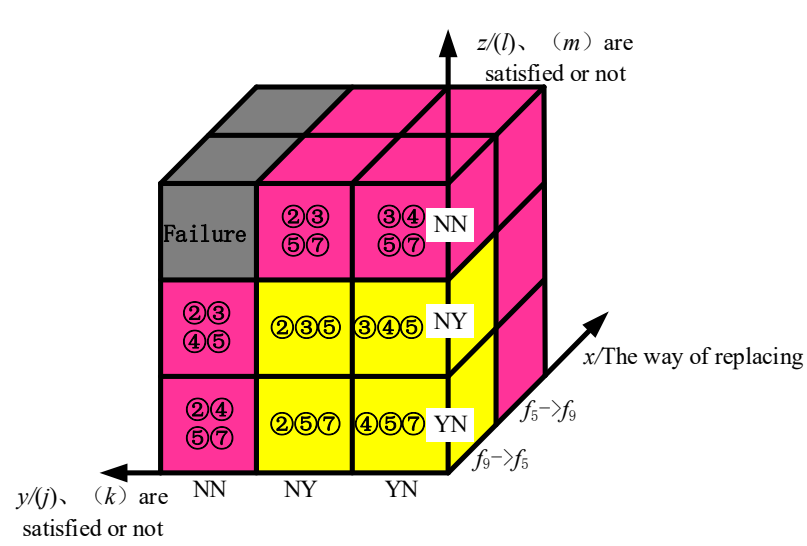

(a) Front view

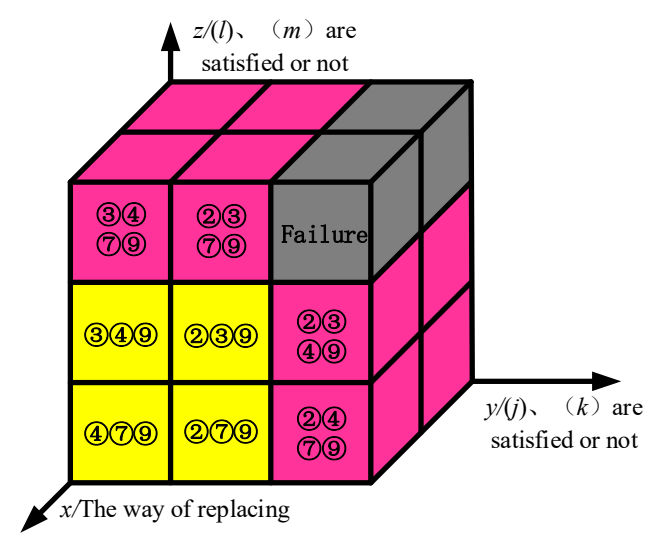

(b) Back view

Fig. 3 Descartes three-dimensional coordinate system of fault diagnosis

Third, because of the importance of the measured values of the branch chain (5) and the branch chain (9) the theoretical values in Eq. (13) use the replacement method to control the variables. On this basis, if there is any measured value matching the theoretical value after replacing the branch chain (5) by the branch chain (9), it does not only explain that the branch chain (9) is undamaged, but also that the branch chain (5) broke down and vice versa.

For the convenience of description, divide Fig. 3 (b) into three layers from bottom to top, parallel to the $\mathrm{x}-\mathrm{y}$ plane, marked as Layer1, Layer2 and Layer3. Next, divide Fig. 3(b) into three columns from left to right parallel to the $\mathrm{x}-\mathrm{z}$ plane, marked as Column1, Column2 and Column3. Further divide Fig. 3(b) into two rows from inside to outside parallel to the $y-z$ plane, marked as Row1, Row2. For example, calculate the force of the branch chain (9) with the force of the branch chain (5) and put the results into $(l),(m)$ in Eq. (13) to calculate the 
theoretical values of the branch chains (3) and (7), which correspond to Row1 in Fig. 3 (b). Then determine whether Eq. $(j)$, Eq. $(k)$,Eq. $(l)$, and Eq. $(m)$ are satisfied. If Eq. $(j)$ is established while Eq. $(k)$ Eq. $(l)$, Eq. $(m)$ are not, Fig. 3 corresponds to Column3 of Layer3, which shows that the branch chains (3) (4) (5) 7 are faulty. If Eq. $(j)$ and Eq. $(l)$ are established while Eq. $(k)$ and Eq. $(m)$ are not, Fig. 3 corresponds to Column2 of Layer2, which shows that the branch chains (2) (3) (5) are faulty. If Eq.(j), Eq. $(k)$, Eq. $(l)$ and Eq. $(m)$ are not established, Fig. 3 corresponds to Column1 of Layer3, which shows the inability to diagnose or the system failure.

Based on the above judgment method, a total of 16 kinds of fault conditions shown in Fig. 3 can be diagnosed in the case of the example.

Obviously, using the Descartes three-dimensional coordinate system of fault diagnosis, the completion of the fault diagnosis of the system only requires quick determination of whether a few equations are satisfied and then finding the corresponding coordinate system cube and reading the corresponding fault branch chains. Rapid diagnosis function can be achieved at the same time.

\subsection{Fault restoration}

After determining specific fault branch chains, it is practicable to carry out the restoration by replacing the fault branch chains according to Eqs. (11), (12) and (13). For example, if it is determined that the fault branch chains are the branch chains (2), (3) and (9), the restoration scheme is shown in the following equations:

$$
\left\{\begin{array}{l}
\hat{f}_{2} \approx f_{4}-\frac{2 m n}{3} \cdot \varepsilon_{x}^{*} \\
\hat{f}_{3} \approx \frac{2 m n}{3} \cdot \varepsilon_{y}^{*}+f_{7} \\
\hat{f}_{9} \approx-k_{9}\left(\frac{f_{5}}{k_{5}}+\frac{f_{6}}{k_{6}}+\frac{f_{8}}{k_{8}}\right)
\end{array}\right.
$$

where $\hat{f}_{2}, \hat{f}_{3}$, and $\hat{f}_{9}$ are the restoration values of the branch chains (2), (3) and (9), respectively.

Put the new values of $\hat{f}_{2}, \hat{f}_{3}$, and $\hat{f}_{9}$ into Eqs. (3) and (4) to calculate the six dimensional acceleration and ensure that the measurement system works normally. Meantime, a six-axis accelerometer model with standard branch chains (2), (3) and (9) is simulated in the Adams software. A comparison chart of the $\mathrm{x}$ linear acceleration measurement precision and theoretical precision after the restoration of the system is shown in Fig. 4. The blue solid line presents the $\mathrm{x}$ linear acceleration after the restoration and the red dotted line shows the $\mathrm{x}$ linear acceleration in Adams. Obviously, the blue solid line and the red dotted line overlap, which shows that the calculated acceleration value is very consistent with the theoretical value obtained by the simulation. On this basis, the error between them can be calculated after the restoration, which is plotted in green dash lines in Fig. 4. As shown in Fig. 4, the maximum value of the absolute error is only $0.1 \%$ although it gradually increases with time. It can satisfy the demand of the practical application of the six-axis accelerometer, which indicates the effectiveness of the restoration. 


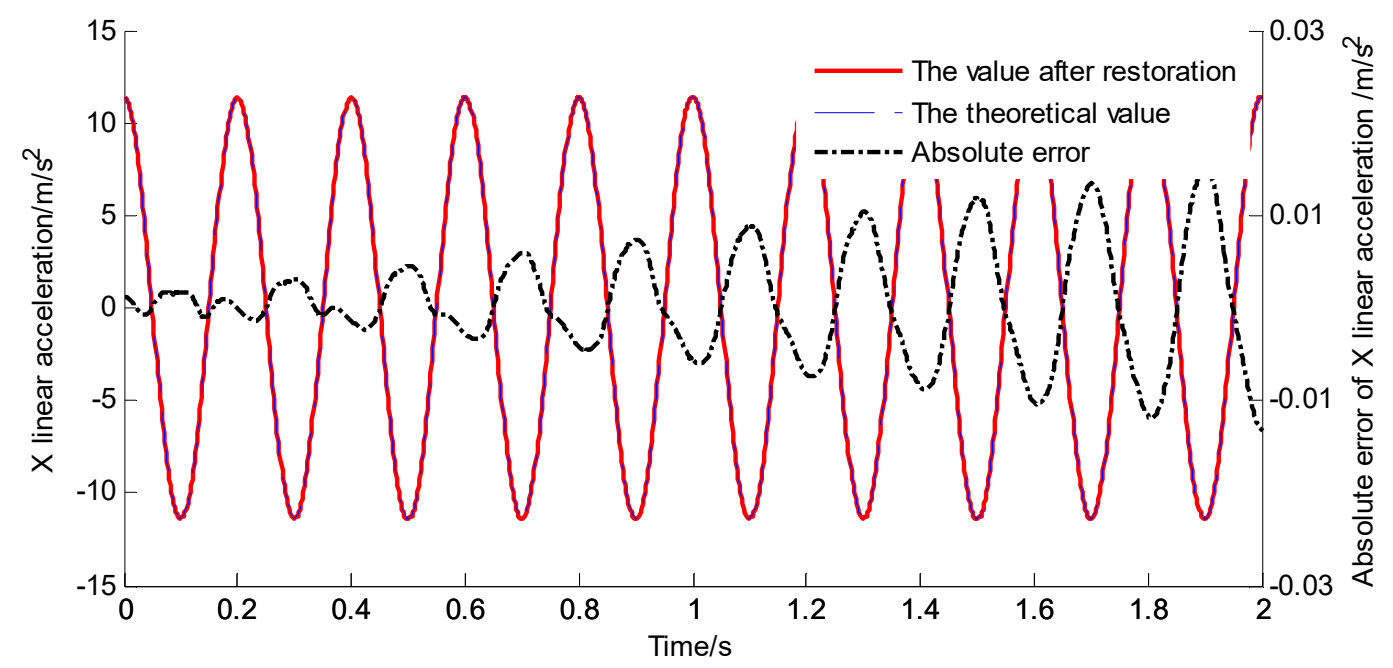

Fig. $4 \mathrm{X}$ linear acceleration measurement precision and theoretical precision after restoration of the system

In the same way, classify and refine possible fault conditions of the measurement system with the gyroscope according to the established conditions of Eqs. (11), (10), (12) and (13); draw the corresponding Descartes three-dimensional coordinate system of fault diagnosis, and improve and complete the fault diagnosis system of the measurement system.

According to the analysis results to conduct a comprehensive statistical analysis and analysis of possible fault conditions of the gyroscope-based six-axis accelerometer, the making of the fault diagnosis of the measurement system only requires the building of 5 Descartes three-dimensional coordinate systems of fault diagnosis as shown in Fig. 3. The statistical analysis shows that there are 165 kinds of fault conditions in total that can be restored in the above mentioned way. They include all fault conditions in a total of $9\left(C_{9}^{1}\right)$ when only one branch chain breaks down, all fault conditions in a total of $36\left(C_{9}^{2}\right)$ when only two branch chains break down, all fault conditions in a total of $84\left(C_{9}^{3}\right)$ when three branch chains break down, and some fault conditions in a total of 36 when four branch chains break down. Table 1 shows the 36 fault conditions can be restored when four branch chains break down. The circled number combination is the faulty branch chain combination.

Table 1 Fault conditions can be restored only when four branch chains break down

\begin{tabular}{|c|c|c|c|c|c|}
\hline \multicolumn{6}{|c|}{ Recoverable conditions } \\
\hline 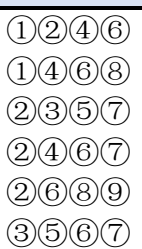 & 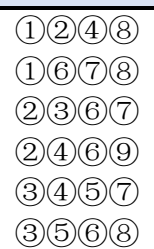 & 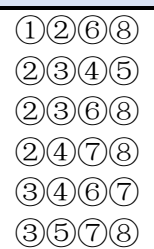 & 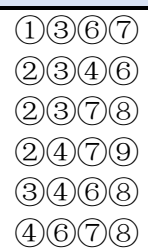 & 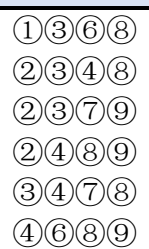 & $\begin{array}{l}1(3)(7) \\
(2)(3)(4) \\
(2)(4) \\
(2) 67 \\
(3)(4) \\
(5)(6)\end{array}$ \\
\hline
\end{tabular}

\section{Experimental setup}

We carried out the tests on the experimental platform of the six-axis accelerometer including some devices shown in Fig. 5. The platform is composed of two test plates, two crank-slider mechanisms, two gears and a rack. Every plate is driven by an AC motor at a constant velocity. The six-axis accelerometer fixed to the gear 1 will rotate synchronously together with the gyroscope fixed to the gear 2 when the crank-slider mechanism 1 is driven and 2 remains static. The rotational velocities of gear 1 and 2 will be measured by the gyroscope. The linear acceleration of the six-axis accelerometer will be measured by the reference device (GINS100) fixed to the plate 2 when the crank-slider mechanism 2 is driven. The properties of the gyroscope (CRS03-04S) and the reference device are shown in Table 2. 


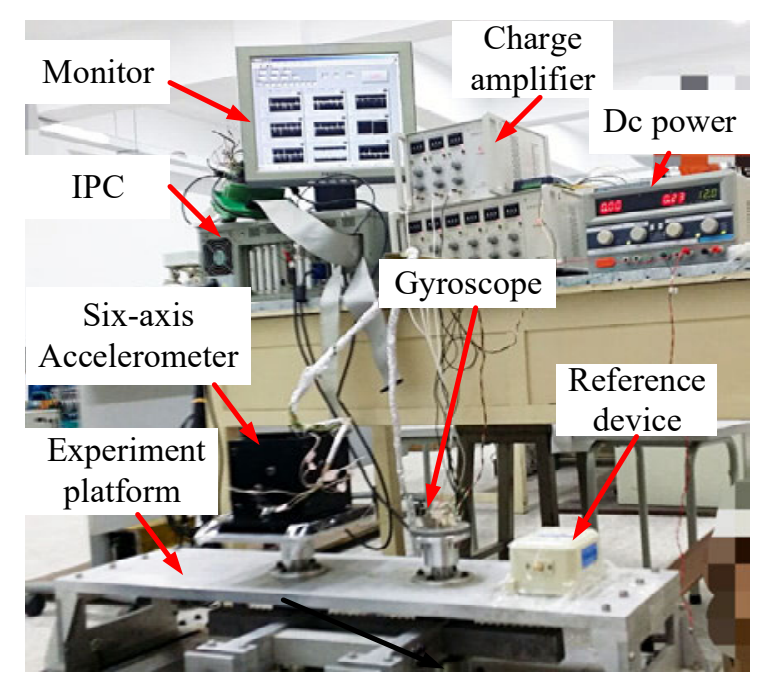

(a) Experimental platform

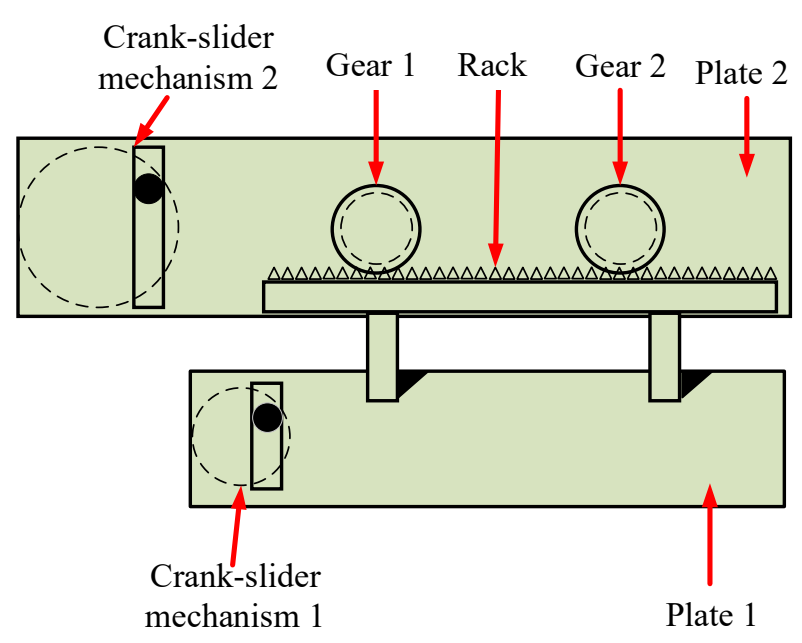

(b) Scheme of the platform

Fig. 5 Experimental platform of six-axis accelerometer

Table 2 Key features of the gyroscope and the reference device

\begin{tabular}{|c|c|c|c|c|c|c|}
\hline Sensors & $\begin{array}{c}\text { Number } \\
\text { of axes }\end{array}$ & $\begin{array}{l}\text { Angular } \\
\text { range }\end{array}$ & Nonlinearity & Drift vs. time & Mass & $\begin{array}{c}\text { Acceleration } \\
\text { range }\end{array}$ \\
\hline CRS03-04S & 3 & $\pm 200^{\circ} / \mathrm{s}$ & $< \pm 0.5 \%$ & $\begin{array}{c}< \pm 0.55^{\circ} / \mathrm{s} \text { in any } \\
30 \mathrm{~s} \text { period }\end{array}$ & $<18$ gram & 1 \\
\hline GINS 100 & 6 & $\pm 250^{\circ} / \mathrm{s}$ & $< \pm 0.5 \%$ & $<12^{\circ} / \mathrm{h}$ & $<500$ gram & $\pm 6 \mathrm{~g}$ \\
\hline
\end{tabular}

When the six-axis accelerometer was driven by the AC motor below the crank-slider mechanism 1 periodically at a constant angular velocity of 200rpm, we collected data of all branch chains and carried out the function of filtering to eliminate outliers. For example, the contrast lines of the branch chain (4) before and after filtering are shown in Fig. 6. In the same way, the filtered forces of each branch chain are shown in Fig. 7.

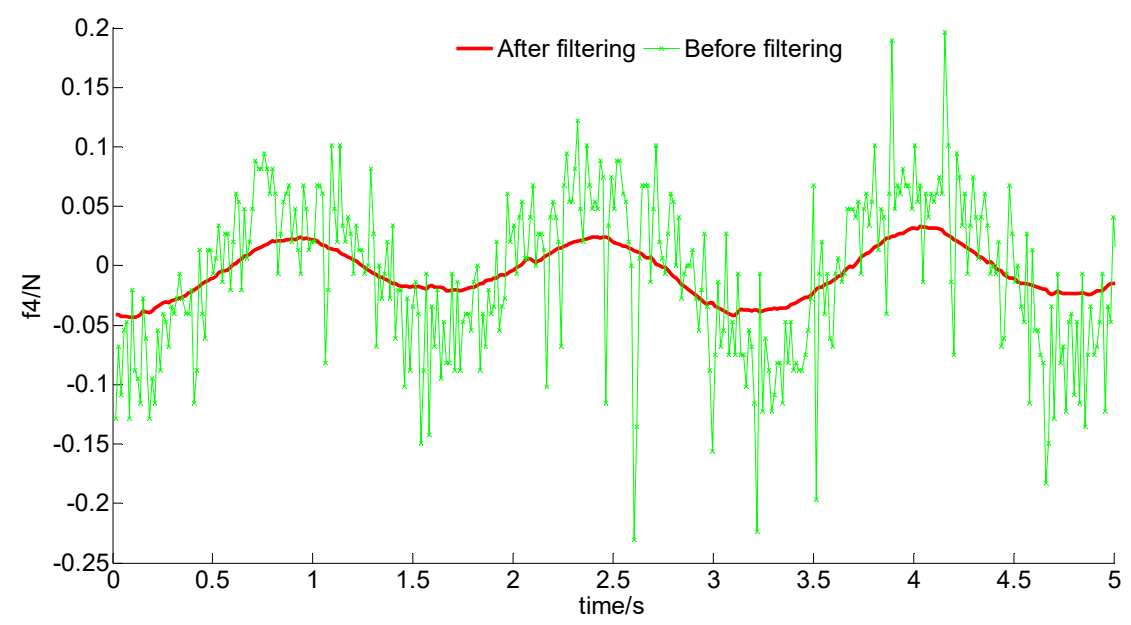

Fig. 6 Comparison of branch chain (4) before and after filtering 

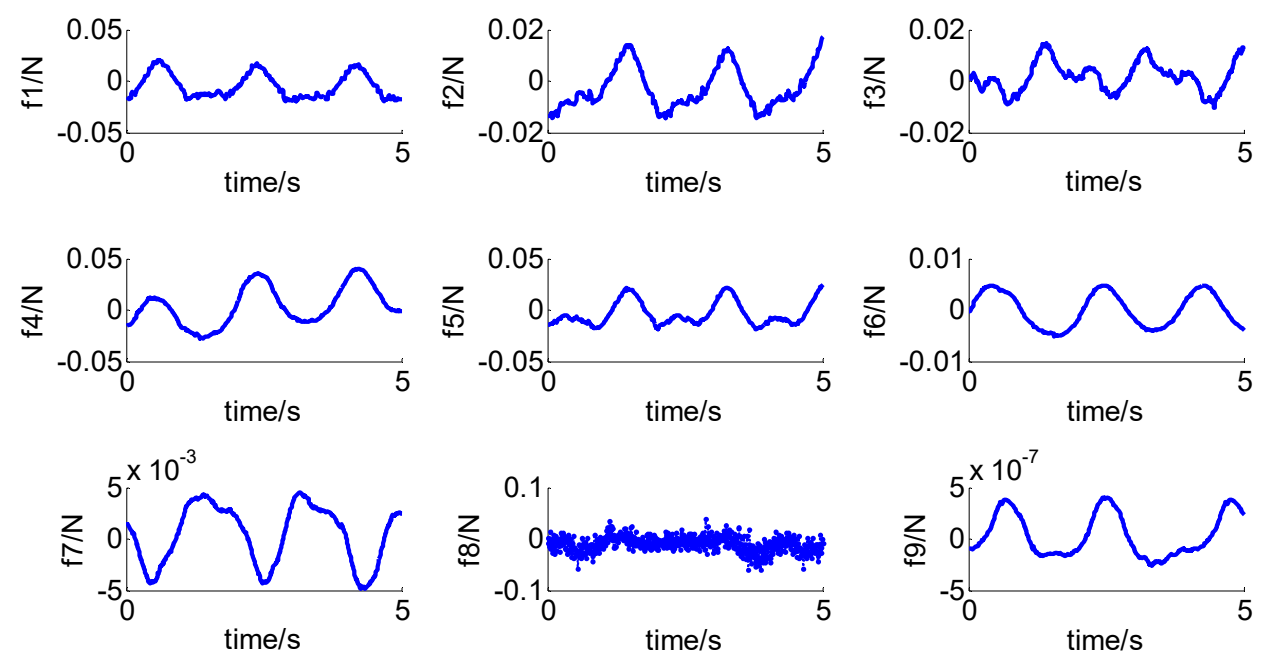

Fig. 7 Forces of each branch chain after filtering

According to the decoupling algorithm presented in section 2.1, accelerations were calculated and compared with the reference device output. For example, the comparison chart of the angular acceleration in the y direction is shown in Fig. 8. Apparently, the measurement results do not agree with the contrast device output, so it is necessary to conduct a fault diagnosis and restoration to correct the error. According to the principle of the fault diagnosis presented above, it is easy to find that Eq.(f), Eq.(i) and Eq. $(o)$ are not established, which explains a fault occurring in the branch (8). The comparison chart of the y linear acceleration measurement precision and the theoretical precision of the system after replacing the branch (8) according to Eq.(o) is shown in Fig. 9.

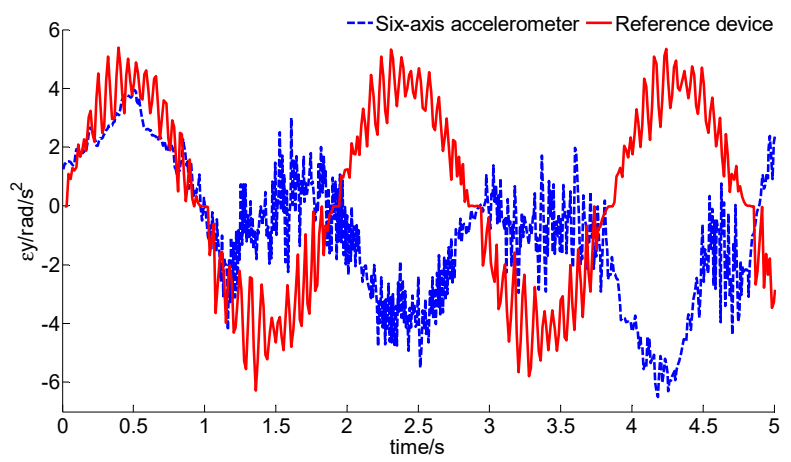

Fig. 8 Comparison chart of angular acceleration of y direction before restoration

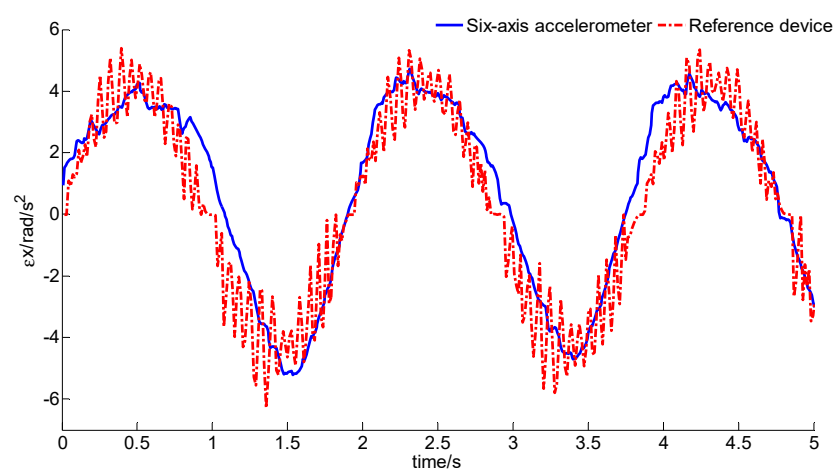

Fig. 9 Comparison chart of angular acceleration in y direction after restoration 
After diagnosis and restoration, the calculation shows that the maximum relative error of the six-axis accelerometer is $31.69 \%$ and the average relative error is $11.53 \%$. The six-axis accelerometer goes back to the measuring state in which the error will be less compared to the original one in which the fault occurs.

\section{Conclusions}

(1) By introducing an auxiliary angular velocity, the dynamic equations of the six-axis accelerometer are completely decoupled. By using the forward kinematics theory of parallel mechanism, we have explored the inherent constraint relations between system inputs and built a theory for the fault diagnosis.

(2) with a gyroscope introduced, the inherent relationship between the inputs of the gyroscope and the six-axis accelerometer are derived, and the basis for fault diagnosis and restoration is obtained. With the aid of the Descartes three-dimensional coordinate system of fault diagnosis, the real-time diagnosis function of the measurement system is achieved.

(3) After determining specific faulty branch chains, data restoration is carried out by replacing the faulty branch chains according to the inherent relationship between the inputs of the gyroscope and the six-axis accelerometer. The simulation analysis of ADAMS shows that the maximum error is only $0.1 \%$. The experimental results are in agreement with the feasibility of the fault restoration scheme.

\section{Acknowledgements}

This research is supported by the National Natural Science Foundation of China (grant no. 51175263) and the Natural Science Foundation of Jiangsu Province (Grant No. BK20141414).

\section{REFERENCES}

[1] Staufer, Peter, and H. Gattringer. "State estimation on flexible robots using accelerometers and angular rate sensors." Mechatronics22.8(2012):1043-1049.

[2] Regal, Cindy. "Sensing nanomechanical motion with a microwave cavity interferometer." Nature Physics 4.7(2008):555-560.

[3] Huang, Yann Chang, H. T. Yang, and C. L. Huang. "Developing a new transformer fault diagnosis system through evolutionary fuzzy logic." Power Delivery IEEE Transactions on 12.2(1997):761-767. https://doi.org/10.1109/61.584363

[4] Z Zhao, Zhi Gang, and W. Zhao. "A New Sensor Failure Detection Method Based on Dynamic Uncertainty Theory." Chinese Journal of Sensors \& Actuators 19.6(2006):2723-2726. (in Chinese)

[5] Fraher, P. M. A., and D. W. Clarke. "Fouling detection and compensation in Clark-type DOx sensors." IEEE Transactions on Instrumentation \& Measurement 47.3(1998):686-691. https://doi.org/10.1109/19.744325

[6] Niaki, Seyed Taghi Akhavan, and B. Abbasi. "Fault Diagnosis in Multivariate Control Charts Using Artificial Neural Networks." Quality \& Reliability Engineering 21.8(2010):825-840. https://doi.org/10.1002/qre.689

[7] Wu, Jian Da, and C. H. Liu. "An expert system for fault diagnosis in internal combustion engines using wavelet packet transform and neural network." Expert Systems with Applications 36.3(2009):4278-4286. https://doi.org/10.1016/j.eswa.2008.03.008

[8] Mayagoitia, R. E., A. V. Nene, and P. H. Veltink. "Accelerometer and rate gyroscope measurement of kinematics: an inexpensive alternative to optical motion analysis systems." Journal of Biomechanics35.4(2002):537-542. https://doi.org/10.1016/S0021-9290(01)00231-7

[9] You, Jing Jing, L. I. Cheng-Gang, and W. U. Hong-Tao. "Study on the scheme design and modeling of six-axis accelerometer based on parallel mechanism." Zhendong Gongcheng Xuebao/journal of Vibration Engineering 25.6(2012):658-666. 
[10] Chablat, D, and P. Wenger. "Architecture optimization of a 3-DOF translational parallel mechanism for machining applications, the orthoglide." Robotics \& Automation IEEE Transactions on 19.3(2007):403410.

[11] Newcomb, C. V., and I. Flinn. "Improving the linearity of piezoelectric ceramic actuators." Electronics Letters 18.11(1982):442-444. https://doi.org/10.1049/el:19820301

[12] Gal-Chen, Tzvi, and R. C. J. Somerville. "On the use of a coordinate transformation for the solution of the Navier-Stokes equations." Journal of Computational Physics 17.2(1975):209-228. https://doi.org/10.1016/0021-9991(75)90037-6

[13] Fang, Y., et al. "Nonlinear coupling control laws for an underactuated overhead crane system." IEEE/ASME Transactions on Mechatronics8.3(2003):418-423. https://doi.org/10.1109/TMECH.2003.816822

[14] Batina, John T. "Unsteady Euler algorithm with unstructured dynamic mesh for complex-aircraft aerodynamic analysis." 29.3(2012):327-333.

[15] Lee, Tae Young, and J. K. Shim. "Forward kinematics of the general 6-6 Stewart platform using algebraic elimination." Mechanism \& Machine Theory 36.9(2001):1073-1085. https://doi.org/10.1016/S0094-114X(01)00034-9

[16] Mboup, Mamadou, C. Join, and M. Fliess. "Numerical differentiation with annihilators in noisy environment." Numerical Algorithms 50.4(2009):439-467. https://doi.org/10.1007/s11075-008-9236-1

[17] Zhang, Hai Li, and H. L. Zhang. "Research and Application of Differential Tracker." Control \& Instruments in Chemical Industry (2013).

Submitted: $\quad$ 02.3.2017

Accepted: $\quad$ 15.3.2018
Aassociate Prof. Chenggang Li

Yan Wang

Jing Chen

Hemant Rajnathsing

College of Mechanical and Electrical

Engineering

Nanjing University of Aeronautics and Astronautics

29 Yudao str., Nanjing 210016, China

E-mail: lichenggang@nuaa.edu.cn

Jingjing You

College of Mechanical and Electronic

Engineering

Nanjing Forestry University

159 Lonpan road, Nanjing 210037, China 